\title{
CD133 Expression as a Helicobacter pylori-independent Biomarker of Gastric Cancer Progression
}

\author{
RACHEL HOWARD $^{1}$, SAMEER AL DIFFALHA ${ }^{2}$, JOSE PIMIENTO ${ }^{3}$, JAIME MEJIA ${ }^{4}$, \\ HEIKO ENDERLING ${ }^{5}$, ANNA GIULIANO ${ }^{6}$ and DOMENICO COPPOLA ${ }^{2}$ \\ ${ }^{1}$ Department of Epidemiology, H. Lee Moffitt Cancer Center \& Research Institute, Tampa, FL, U.S.A.; \\ ${ }^{2}$ Pathology, H. Lee Moffitt Cancer Center \& Research Institute, Tampa, FL, U.S.A.; \\ ${ }^{3}$ Surgical Oncology, H. Lee Moffitt Cancer Center \& Research Institute, Tampa, FL, U.S.A.; \\ ${ }^{4}$ Department of Pathology, Instituto de Patología Mejía Jiménez in Cali, Pathology, Valle del Cauca, Colombia; \\ ${ }^{5}$ Integrated Mathematical Oncology, H. Lee Moffitt Cancer Center \& Research Institute, Tampa, FL, U.S.A.; \\ ${ }^{6}$ Center for Infection Research in Cancer, H. Lee Moffitt Cancer Center \& Research Institute, Tampa, FL, U.S.A.
}

\begin{abstract}
Background/Aim: Gastric adenocarcinoma is the fourth most common cancer worldwide. While gastric cancer prevalence varies globally and incidence rates are decreasing in the West, many cases continue to be diagnosed at an advanced stage and the 5-year survival rate still falls below 30\%. Early treatment of gastric cancer by endoscopic and/or surgical therapy may decrease mortality; yet reliable, universally applicable biomarkers for early detection of gastric cancer have still not been established. Materials and Methods: The present work compares the expression of CD133 (prominin-1), a potential biomarker of disease progression in gastric cancer, between independent cohorts of $H$. pylori $(+)$ and $H$. pylori (-) patients at each respective stage of carcinogenesis. H. pylori (-) patients $(N=45)$ who underwent gastric biopsy at the Moffitt Cancer Center (MCC) in Tampa, Florida, and H. pylori $(+)$ patients $(N=59)$ who underwent gastric biopsy at the Instituto de Patologia Mejia Jimenez (IPMJ) in Cali, Colombia were evaluated and immunostained for CD133. Results: A statistically significant increase in CD133 expression (in terms of the Allred score) was observed between all stages of progression (normal mucosa, inflammation/metaplasia, low-grade dysplasia and gastric adenocarcinoma) for each respective patient cohort. No statistically significant difference in CD133 expression at each respective stage of disease was observed between the
\end{abstract}

Correspondence to: Domenico Coppola, MD, Professor of Pathology and Tumor Biology, Department of Anatomic Pathology, Moffitt Cancer Center and Research Institute University of South Florida, College of Medicine, Tampa, FL, U.S.A. Tel: +1 8137453275, Fax: +1 8137451708, e-mail: domenico.coppola@moffitt.org

Key Words: CD133, gastric cancer biomarker, gastric adenocarcinoma, Helicobacter pylori.
H. pylori-positive and negative-cohorts. Conclusion: The observation of distinct stepwise increases in CD133 expression in both patient cohorts, and the lack of any significant difference between groups, suggests that CD133 expression may serve as a biomarker for early detection of gastric cancer independent of bacterial status and strain, and corresponding differences in disease histomorphology and classification. This warrants further validation on larger independent cohorts across multiple geographic regions and incorporating multiple bacterial strain types.

Approximately one million patients are diagnosed with gastric adenocarcinoma (GC) every year, making it the second most common cause of cancer-related death worldwide (1-4). While genetic factors, as well as alcohol consumption and diet can play an important role in the etiology of GC, infection with Helicobacter pylori remains the most common cause of the disease, associated with over $70 \%$ of GC incidence $(5,6)$. The bacterial infection typically induces distal/non-cardia gastric cancer, classified histomorphologically as intestinal type and characterized by a stepwise progression to cancer featuring $H$. pylori-related chronic active gastritis, atrophy, intestinal metaplasia, lowgrade dysplasia, high-grade dysplasia, intramucosal adenocarcinoma, and invasive adenocarcinoma (7-9).

While $H$. pylori prevalence is particularly high in Africa, South and East Asia and parts of South America, GC incidence still varies significantly due to the diversity of bacterial strains across these regions. The highest rates of $H$. pylori-associated GC incidence and mortality are found in regions where the $\mathrm{CagA}+\mathrm{VacA}+$ strain is prevalent, including Japan and Colombia (10). In these regions, effective screening is crucial to reducing the high mortality rate, and the identification of reliable biomarkers for early detection continues to be at the forefront of such efforts. 
Table I. Baseline patient characteristics for the two cohorts: Moffitt Cancer Center, Tampa, Florida (N=48) and Instituto de Patologia Mejia Jimenez, Cali, Colombia (N=58).

\begin{tabular}{|c|c|c|c|c|c|c|c|c|}
\hline \multirow{2}{*}{$\begin{array}{l}\text { Cohorts } \\
\text { Stage }\end{array}$} & \multicolumn{4}{|c|}{ H. pylori (-) (Tampa, Florida) } & \multicolumn{4}{|c|}{ H. pylori (+) (Cali, Colombia) } \\
\hline & NM & $\mathrm{IM}$ & DS & GC & NM & $\mathrm{IM}$ & DS & GC \\
\hline $\mathrm{N} 1$ & 1 & 9 & 9 & 19 & 10 & 17 & 10 & 21 \\
\hline Age & $66( \pm 16)$ & $58( \pm 14)$ & $66( \pm 12)$ & $69( \pm 13)$ & $43( \pm 11)$ & $62( \pm 16)$ & $52( \pm 16)$ & $53( \pm 14)$ \\
\hline \multicolumn{9}{|l|}{ Gender } \\
\hline Male & $64 \%$ & $89 \%$ & $78 \%$ & $84 \%$ & $90 \%$ & $53 \%$ & $30 \%$ & $52 \%$ \\
\hline Female & $36 \%$ & $11 \%$ & $22 \%$ & $16 \%$ & $10 \%$ & $47 \%$ & $70 \%$ & $48 \%$ \\
\hline
\end{tabular}

NM, Normal gastric mucosa; IM, inflammation or metaplasia; DS, low-grade dysplasia; GC, adenocarcinoma.

CD133 (prominin-1) is a 120-kDa penta-span membrane glycoprotein product of the CD133 gene located on chromosome $4(4 \mathrm{p} 15.33)$ and is localized to the microvilli and other protrusions of the plasma membrane (11). In recent years the role of CD133 as a prognostic marker in gastric cancer, as well as in other cancers including those of the colon, pancreas, lung, liver and ovary, has been widely studied (12-18). In gastric cancer, several lines of research have shown that overexpression of CD133 is associated with later TNM stage, lymph node metastasis, chemo-resistance, early recurrence, lymphatic and vascular invasion, and worse prognosis (19-25). The level of CD133 mRNA expression may also reflect the status of lymph node metastasis as well as tumor size and stage (26). The positive rate of CD133 immunostain has been approximated at $35.1 \%$ of GC cases in a recent meta-analysis (19). While the relationship between CD133 expression in GC and patient outcomes has been relatively widely studied, a stepwise increase in CD133 during the earlier stages of disease has also recently been proposed (27-29). To date, existing studies have either associated this stepwise increase in CD133 expression with the H. pylori-driven Correa pathway of carcinogenesis, or have not investigated the relationship between expression and infection status.

In this manuscript we evaluated whether a progressive increase in CD133 expression during carcinogenesis is specific to $H$. pylori-associated disease by comparing expression at each respective stage of the Correa pathway between independent patient cohorts from Cali, Colombia (H. pylori (+) patients) and Tampa, Florida (H. pylori (-) patients). A stepwise increase in $\mathrm{CD} 133$ positivity between the respective stages of normal gastric mucosa, inflammation or metaplasia, low-grade dysplasia, and gastric adenocarcinoma was observed in both $(H$. pylori $(+)$ and $H$. pylori $(-))$ patient cohorts, with no significant difference between the two cohorts at each stage of progression. This preliminary result challenges the previous assumption that $\mathrm{CD} 133$ expression is specific to the bacteria-induced Correa pathway, and suggests that CD133 could serve as independent biomarker for gastric cancer progression, irrespective of disease etiology or histomorphological disease classification.

\section{Materials and Methods}

Patients and samples. Samples were obtained retrospectively from 45 H. pylori (-) patients who underwent gastric biopsy at the Moffitt Cancer Center (MCC) in Tampa, Florida during 2015 and 2016, and 59 H. pylori (+) patients who underwent gastric biopsy at the Instituto de Patologia Mejia Jimenez (IPMJ) in Cali, Colombia during 2014. Biopsy samples were subclassified based on the pathological analysis of histologic lesions according to the four different stages of the Correa pathway (30): normal gastric mucosa (NM), inflammation or metaplasia (IM), low-grade dysplasia (DS) and adenocarcinoma (GC). Inflammation and metaplasia were combined into one category for comparison of the two cohorts due to the inherent differences in disease development between bacteriaassociated and non-associated disease. Stage of progression was assessed by hematoxylin and eosin (H\&E) staining, and H. pylori status was evaluated by immunohistochemical staining of endoscopy samples from each patient.

The southern United States is a particularly low H. pylori incidence region; if the infection does occur, the bacterial strain is typically CagA-/VacA- and un-associated with the onset of carcinogenesis. GC patients from Tampa, Florida primarily presented with diffuse/cardia cancers. Comparably, the mountainous region of Cali, Colombia has extremely high $(>70 \%)$ prevalence of CagA+/VacA+ H. pylori and patients typically presented with intestinal-type cancers of the antrum and corpus. An overview of patient characteristics is provided in Table I. Of the MCC patients, $73 \%$ were Caucasian, 15\% Hispanic, and $13 \%$ Asian. All patients from IPMJ were Hispanic. All information linked to the patients is protected and de-identified, and all tissue samples are collected from retrospective cases; informed consent or substitute was obtained from all patients for future use of clinical data and samples. All specimens were de-identified by the IRB at the Instituto de Patología Mejía Jiménez in Cali, Columbia, USA, both for the initial selection of the samples and for the subsequent data collection.

Immunohistochemistry. All Cali samples were shipped as formalinfixed paraffin-embedded (FFPE) tissue blocks to Moffitt Cancer Center, Tampa, FL for immunohistochemical analysis, as 
previously described (31). All samples from both cohorts were stained using the Ventana automated immunostainer Discovery XT (Ventana, Tucson, AZ, USA) and Ventana ChromoMap kit (Ventana). Tissue sections of $4 \mu \mathrm{m}$ were incubated for $2 \mathrm{~h}$ with mouse anti-human CD133 monoclonal antibody (1:100; MAB4399, Millipore, Billerica, MA, USA), followed by incubation with the Ventana OmniMap anti-mouse secondary antibody (Ventana) for 16 min. Kidney tissue samples were used as the positive control, while ommission of the first antibody served as negative control. Slides were counterstained with $\mathrm{H} \& \mathrm{E}$. All sections were dehydrated and coverslipped as per normal laboratory protocol. Slides were read by two pathologists. The immunostain for CD133 was localized to the cytoplasm in all cases. At each disease stage, extent of immunohistochemical staining (of epithelial cells) in terms of both percentage staining and staining intensity was evaluated. The Allred scoring system featuring a proportion score (scale of 0-5) and an intensity score (scale of $0-3$ ) was utilized to give a total positivity score between 0 and 8 (32).

Statistical analysis. The statistical significance of the difference in CD133 positivity score between stages (e.g., normal mucosa vs. inflammation/metaplasia, inflammation/metaplasia $v s$. low-grade dysplasia, and low-grade dysplasia $v s$. carcinoma) was evaluated by two-sample t-test and (non-parametric) Wilcoxon rank sum test for the $H$. pylori (-) cohort. Results were then compared in a similar manner to equivalent increases in CD133 expression in the H.pylori (+) cohort to evaluate the influence of disease etiology and pathology on marker expression. All $p$-values $<0.05$ were considered statistical significant.

\section{Results}

In the H.pylori (-) cohort, the CD133 IHC total positivity score (TPS) was significantly higher in both DS (TPS increase $117 \%, p=0.0001$ ) and GC (TPS increase $152 \%, p=0.00001$ ) than in NM (Figure 1). The TPS increase between NM and IM was not statistically significant. A significant increase in CD133 TPS was also observed between the IM and DS samples (TPS increase 52\%, $p=0.012$ ) and the DC and GC samples (TPS increase $16 \%, p=0.03$ ).

In the $H$. pylori (+) cohort, the TPS increase was significant for all stages of disease compared to NM: IM samples (TPS increase 65\%, $p=0.02$ ), DC samples (TPS increase $109 \%, p=0.001$ ), and GC samples (TPS increase $139 \%, p=0.0001$ ) (Figures 1 and 2). A significant increase in CD133 TPS was also observed between the IM and DC samples (TPS increase 26\%, $p=0.003$ ) and the DC and GC samples (TPS increase $15 \%, p=0.045$ ).

Interestingly, no statistically significant difference in CD133 TPS at each respective stage of disease was observed between the $H$. pylori positive and negative cohorts. With an average difference of less than $6 \%$ between the two cohorts, and a minimum $p$-value of 0.23 (GC samples, Tampa $v s$. Cali), the current study demonstrates no evidence that the CD133 increase during gastric carcinogenesis is specific to H. pylori-associated disease.

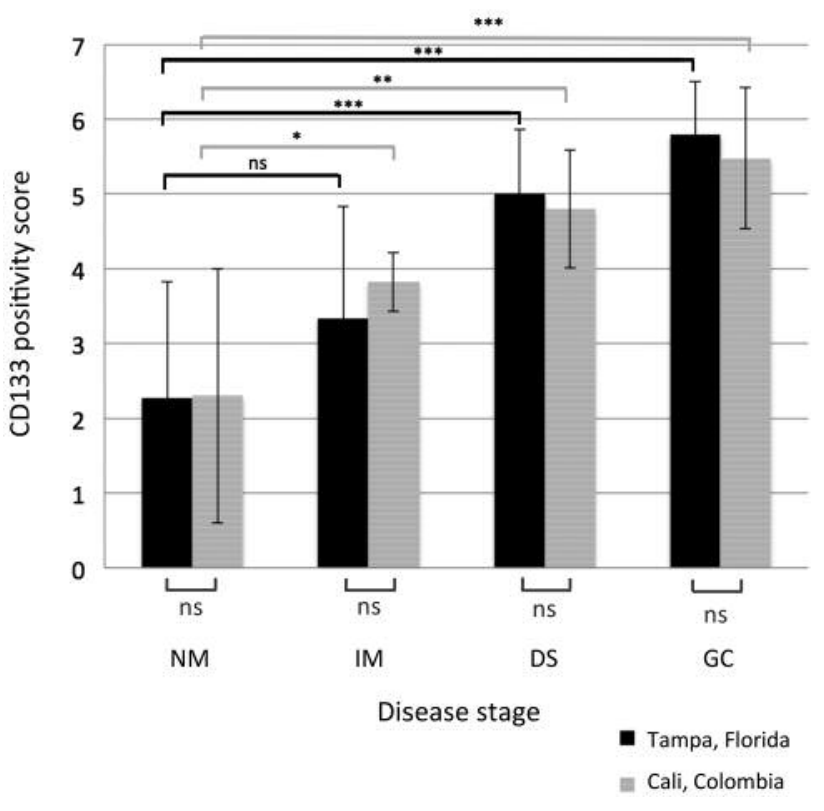

Figure 1. Total CD133 positivity score (Allred scoring system) for each respective patient cohort at each stage of disease. In both cohorts, a statistically significant increase in CD133 expression was observed between all stages of progression, with only one exception of normal mucosa (NM) to inflammation/metaplasia (IM) in the H. pylori negative (Tampa, Florida) group $(* p<0.05, * * p<0.001, * * * p<0.0001)$. No significant difference in CD133 expression was observed between the $H$. pylori-positive and $H$. pylori-negative cohorts at any point in the progression pathway. DS, Lowgrade dysplasia; GC, gastric adenocarcinoma; $n$, non significant.

Tests were repeated using both the percentage score component of the Allred TPS alone and the intensity score component alone to confirm that results are not disproportionally skewed. No statistically significant difference between the $H$. pylori positive and negative cohorts was observed in either of these subcomponents of the TPS.

It was also observed that the CD133 stain localization was usually luminal in better differentiated GC and became cytoplasmic in less differentiated GC tumors (Figure 3). Variations in the localization of CD133 staining were unrelated to the $H$. pylori status.

\section{Discussion}

GC has a high rate of recurrence and metastasis, and typically responds poorly to therapy (34). While the molecular mechanisms responsible for this behavior are still not fully elucidated, elevated CD133 expression has been linked to several of these traits. Yiming et al. have reported a meta-analysis of 8 studies, which included 603 cases of gastric cancer, and have found that CD133 overexpression was associated with higher TNM stage, presence of lymph 


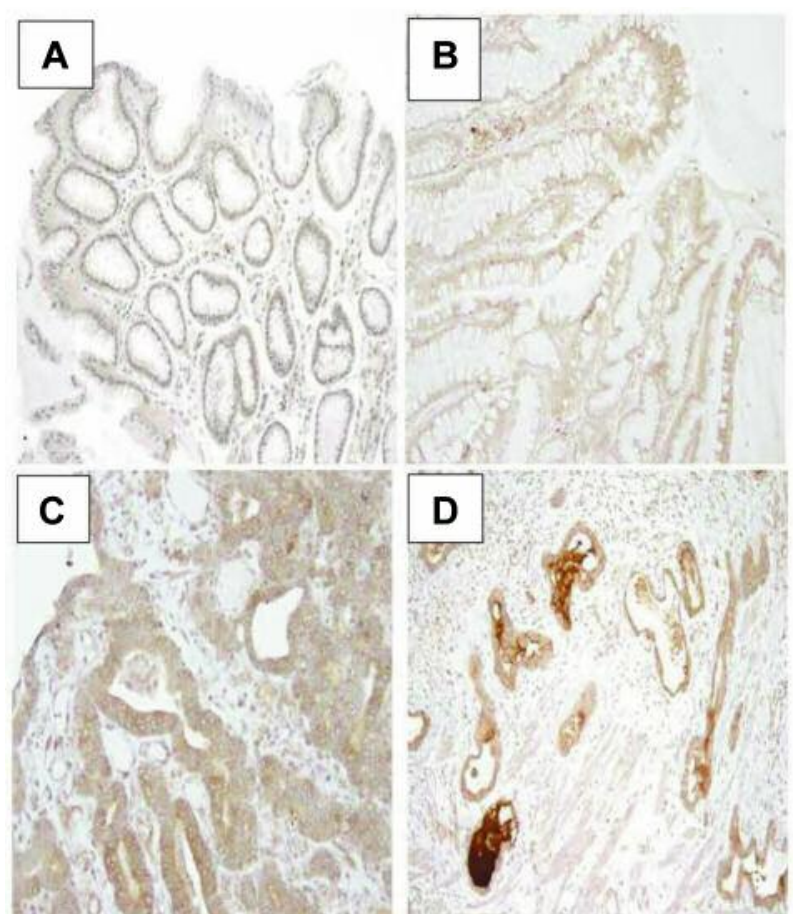

Figure 2. Changes in CD133 immunohistochemical (IHC) expression in different stages of gastric cancer progression. The CD133 IHC staining is negative in the normal gastric mисоsa (A), while it is weakly expressed in intestinal metaplasia (B).CD133 IHC staining is moderate and diffuse in the dysplastic mucosa $(C)$ and strong and diffuse in the gastric adenocarcinoma $(D)$. This same pattern of stain was observed for both H. pylori (+) intestinal-type and H. pylori (-) diffuse-type gastric cancers.
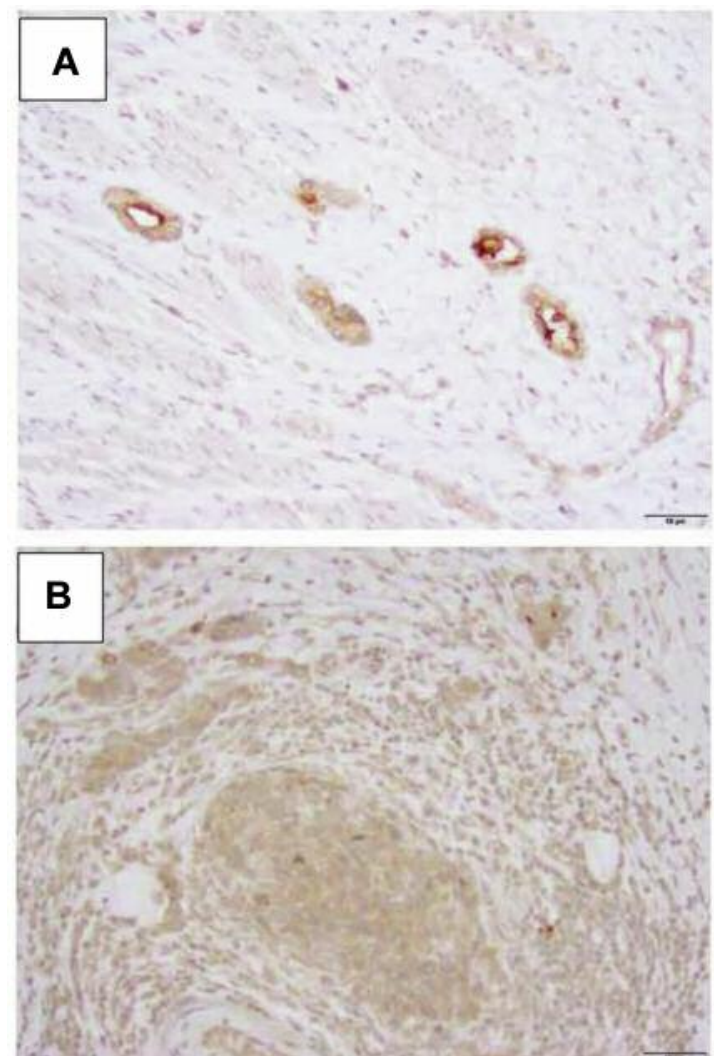

Figure 3. CD133 staining luminal localization seen in a well-differentiated gastric adenocarcinoma $(G C)(A)$ compared to the CD133 cytoplasmic expression observed in a poorly differentiated $G C(B)$. node metastasis, occurrence of vascular and lymphatic invasion and worse prognosis (19). Similarly, Yu et al. have studied 31 gastric cancers comparing CD133 mRNA expression levels in gastric cancer samples to non-cancerous gastric mucosa samples from the same patient (35), showing that the level of CD133 mRNA was associated with the status of cancer, tumor size, tumor stage and presence of metastasis. This finding was is in agreement with the observation of Wen et al. that CD133 mRNA levels were higher in gastric cancer compared to non-neoplastic gastric tissues (20).

While up-regulation of CD133 in high-stage gastric tumors and its association with worse outcomes has already been established, the stepwise increase in CD133 marker positivity during the carcinogenic pathway associated with $H$. pylori is a more recent observation and has highlighted the potential for CD133 expression to act as a marker of disease initiation and progression. Herein, a significant stepwise increase in CD133 expression during the progression of non- $H$. pyloriassociated GC is reported. The absence of a significant difference in CD133 protein expression between cohorts of
$H$. pylori positive and $H$. pylori negative patients at any stage of GC development is also reported.

CD133 has been widely described as a marker of stem cells (SC), particularly in the setting of gastrointestinal (GI) disease. This has been established in multiple cancer types, and an increased SC population has been independently associated with worse prognosis $(20,24,25,27)$. Interestingly, H. pylori has been associated with an increase in the SC population in gastric cancer. This is believed to be attributable to both increased local SC proliferation and recruitment of SCs from the bone marrow in response to persistent bacteria-induced gastritis and tissue atrophy, as well as direct DNA damage and disruption of SC differentiation (36-40). These observations have led to speculation that the previously observed increase in CD133 during $H$. pylori-associated carcinogenesis is attributable to an increasing SC population in the glands of $H$. pylori (+) patients (27). In the absence of the bacteria, however, GC is typically of diffuse-type, localized in closer proximity to the cardia, and is not associated with the clearly defined Correa pathway characterized by intermediate precancerous lesions such as intestinal metaplasia. 
In the present study, the observation that an equivalent increase in CD133(+) SCs occurs in both cancer types, initially led us to speculate that inflammation, common to both $H$. pylori $(+)$ intestinal-type and $H$. pylori (-) diffusetype gastric cancers, could be driving an increase in the SC population during carcinogenesis, irrespective of the bacterial status and the corresponding differences in disease histomorphology and classification. However, our study also showed that although overall marker positivity score increased incrementally during disease progression, expression tended to be of higher intensity in the early stages, becoming of lower intensity - though staining a larger fraction of the cell population - in cancer samples. This appears to be in agreement with recent reports that CD133 is not exclusively a marker of SCs; Ding et al. have suggested that CD133 may also be expressed in transit amplifying progenitor cells of the gastric glands (41). Similar suggestions have been made in other cancer sites, for example it has been calculated that only 1 in 262 CD133 positive cells in colon cancer represents a SC (42). The present work contributes to the expanding network of evidence suggesting caution should be exercised in applying CD133 as a marker of gastric SCs without verification with other common SC markers such as CD44 or Lgr5.

Finally, a change in CD133 localization from the luminal surface to the cytoplasm depending on tumor differentiation was also observed. This finding has previously been reported in other GI tumors and it has been suggested that different localization of CD133 expression may confer different cellular functions $(44,45)$. For example, the apical localization of CD133 may imply that this cholesterolbinding glycoprotein plays a role in the cellular secretory process of well-differentiated tumor cells and that this role is lost in poorly differentiated cells (46).

To summarize, we propose that increases in CD133 protein expression during GC carcinogenesis may be independent of the bacterial status or strain and also independent of the role of CD133 as a SC marker. This motivates prospective analysis of the association of CD133 with $H$. pylori infection on larger cohorts, before conclusions can be made regarding its suitability as a general marker of GC initiation and progression.

Due to the commonly late diagnosis and the associated high mortality of GC, reliable biomarkers of disease initiation and progression are crucial for early intervention and improved outcomes. Standardized screening programs for gastric cancer have been trialed on a limited basis and have shown potential to improve outcomes (43). However, in the absence of reliable biomarkers, wide reaching, efficient screening protocols are a near impossibility despite their potential to ultimately facilitate early intervention by endoscopic and/or surgical therapy, and in turn increase survival rates in GC.

\section{Acknowledgements}

The Authors thank the Histology Laboratory at the Moffitt Cancer Center for performing the immunohistochemical stain.

\section{References}

1 Alberts SR, Cervantes A and van de Velde CJ: Gastric cancer: epidemiology, pathology and treatment. Ann Oncol 14(Suppl 2): ii31-36, 2003.

2 Luo GF, Zhang Y, Guo P, Wang L, Huang Y and Li K: Global patterns and trends in stomach cancer incidence: Age, period and birth cohort analysis. Int J Cancer 141(7): 1333-1344, 2017.

3 Bertuccio P, Chatenoud L, Levi F, Praud D, Ferlay J, Negri E, Malvezzi M and La Vecchia C: Recent patterns in gastric cancer: A global overview. Int J Cancer 125(3): 666-673, 2009.

4 Camargo MC, Anderson WF, King JB, Correa P, Thomas CC, Rosenberg PS, Eheman CR and Rabkin CS: Divergent trends for gastric cancer incidence by anatomical subsite in US adults. Gut 60(12): 1644-1649, 2011

5 Zabaleta J: Multifactorial etiology of gastric cancer. Methods Mol Biol 863: 411-35, 2012.

6 Yang P, Zhou Y, Chen B, Wan HW, Jia GQ, Bai HL and Wu XT: Overweight, obesity and gastric cancer risk: Results from a metaanalysis of cohort studies. Europ J Cancer 45(16): 2867-2873, 2009.

7 Correa P: Helicobacter pylori and gastric cancer: state of the art. Cancer Epidemiol Biomarkers Prev 5(6): 477-481, 1996.

8 Machida-Montani A, Sasazuki S, Inoue M, Natsukawa S, Shaura K, Koizumi Y, Kasuga Y, Hanaoka T and Tsugane S: Association of Helicobacter pylori infection and environmental factors in noncardia gastric cancer in Japan. Gastric Cancer 7(1): 46-53, 2004.

9 Lauren P: The Two Histological Main Types of Gastric Carcinoma: Diffuse and So-Called Intestinal-Type Carcinoma. An Attempt at a Histo-Clinical Classification. Acta Pathol Microbiol Scand 64: 31-49, 1965.

10 Watada M, Shiota S, Matsunari O, Suzuki R, Murakami K, Fujioka T and Yamaoka Y: Association between Helicobacter pylori cagA-related genes and clinical outcomes. J Gastroent Hepatol 27: 2, 2012.

11 Li Z: CD133: a stem cell biomarker and beyond. Exp Hematol Oncol 2(1): 17, 2013.

12 Wang K, Xi J, Zhang J and Huang J: Prognostic role of CD133 expression in colorectal cancer: a meta-analysis. BMC Cancer 12: 573, 2012.

13 Kim HS, Yoo SY, Kim KT, Park JT, Kim HJ and Kim JC: Expression of the stem cell markers CD133 and nestin in pancreatic ductal adenocarcinoma and clinical relevance. Int $\mathbf{J}$ Clin Exp Pathol 5(8): 754-61, 2012.

$14 \mathrm{Li} \mathrm{X}$, Zhao H, Gu J and Zheng L: Prognostic value of cancer stem cell marker CD133 expression in pancreatic ductal adenocarcinoma (PDAC): a systematic review and metaanalysis. Int J Clin Exp Pathol 8(10): 12084-12092, 2015.

15 Wu H, Qi X, Yan G, Zhang Q, Xu C and Bian X: Is CD133 expression a prognostic biomarker of non-small-cell lung cancer? A Systematic Review and Meta-Analysis. Plos One 9(6): e100168, 2014

16 Zhong $\mathrm{C}, \mathrm{Wu}$ JD, Fang MM and Pu LY: Clinicopathological significance and prognostic value of the expression of the cancer stem cell marker CD133 in hepatocellular carcinoma: a metaanalysis. Tumor Biology 36(10): 7623-7630, 2015. 
17 Zhou Q, Chen A, Song H, Tao J, Yang H and Zuo M: Prognostic value of cancer stem cell marker CD133 in ovarian cancer: a meta-analysis. Int J Clin Exp Med 8(3): 3080-3088, 2015.

18 Chen S, Song X, Chen Z, Li X, Li M, Liu H and Li J: CD133 Expression and the Prognosis of Colorectal Cancer: A Systematic Review and Meta-Analysis. Plos One 8(2): e56380, 2013.

19 Yiming L, Yunshan G, Bo M, Yu Z, Tao W, Gengfang L, Dexian F, Shiqian C, Jianli J, Juan T and Zhinan C: CD133 overexpression correlates with clinicopathological features of gastric cancer patients and its impact on survival: a systematic review and meta-analysis. Oncotarget 6(39): 42019-42027, 2015.

20 Wen L, Chen X, Yang K, Chen Z, Zhang B, Chen J, Zhou Z, Mo $\mathrm{X}$ and $\mathrm{Hu} \mathrm{J}$ : Prognostic value of cancer stem cell marker CD133 expression in gastric cancer: a systematic review. PLoS One 8(3): e59154, 2013.

21 Hashimoto K, Aoyagi K, Isobe T, Kouhuji K and Shirouzu K: Expression of CD133 in the cytoplasm is associated with cancer progression and poor prognosis in gastric cancer. Gastric Cancer 17(1): 97-106, 2014.

22 Lee HH, Seo KJ, An CH, Kim JS and Joen HM: CD133 expression is correlated with chemoresistance and early recurrence of gastric cancer. J Surg Oncol 106(8): 999-1004, 2012.

23 Cai C, Yu JW, Wu JG, Lu RQ, Ni XC, Wang SL and Jiang BJ: CD133 promotes the invasion and metastasis of gastric cancer via epithelial-mesenchymal transition. Zhonghua Wei Chang Wai Ke Za Zhi 16(7): 662-667, 2013.

24 Ishigami S, Ueno S, Arigami T, Uchikado Y, Setoyama T, Arima H, Kita Y, Kurahara H, Okomura H, Matsumoto M, Kijima Y and Natsugoe S: Prognostic Impact of CD133 Expression in Gastric Carcinoma. Anticancer Res 30(6): 2453-2457, 2010.

25 Saricanbaz I, Karahacioglu E, Ekinci O, Bora H, Kilic D and Akmansu M: Prognostic Significance of Expression of CD133 and Ki-67 in Gastric Cancer. Asian Pacif J Cancer Prev 15(19): 8215-8219, 2014.

26 Yu JW, Zhang P, Wu JG, Wu SH, Li XQ, Wang ST, Lu RQ, Ni $\mathrm{XC}$ and Jiang BJ: Expressions and clinical significances of CD133 protein and CD133 mRNA in primary lesion of gastric adenocacinoma. J Exp Clin Cancer Res 29: 141, 2010.

27 Wang T, Ong CW, Shi J, Srivastava S, Yan B, Cheng CL, Yong WP, Chan SL, Yeoh KG, Iacopetta B and Salto-Tellez M: Sequential expression of putative stem cell markers in gastric carcinogenesis. Br J Cancer 105(5): 658-665, 2011.

28 Walker R, Poleszczuk J, Mejia J, Lee JK, Pimiento JM, Malafa M, Giuliano AR, Enderling H and Coppola D: Toward early detection of Helicobacter pylori-associated gastric cancer. Gastric Cancer 21(2): 196-203, 2017.

29 Abd El Atti RM and Abu-Zeid RM: CD133 and FGF7: a link between Helicobacter pylori-induced gastritis and gastric carcinoma. Egyptian J Pathology 32(1): 142-149, 2012.

30 Correa P, Piazuelo MB and Wilson KT: Pathology of gastric intestinal metaplasia: clinical implications. Am J Gastroenterol 105(3): 493-8, 2010.

31 Walker R, Mejia J, Lee JK, Pimiento JM, Malafa M, Giuliano AR, Coppola D and Enderling H: Personalizing gastric cancer screening with predictive modeling of disease progression biomarkers. Appl Immunohistochem Mol Morphol, 2017. doi: 10.1097/PAI.0000000000000598.
32 Allred DC, Harvey JM, Berardo M and Clark GM: Prognostic and predictive factors in breast cancer by immunohistochemical analysis. Mod Pathol 11(2): 155-68, 1998.

33 Peichev M, Naiyer AJ, Pereira D, Zhu Z, Lane WJ, Williams M, Oz MC, Hicklin DJ, Witte L, Moore MA and Rafii S: Expression of VEGFR-2 and AC133 by circulating human CD34(+) cells identifies a population of functional endothelial precursors. Blood 95(3): 952-8, 2000.

34 Dikken JL, can de Velde CJH, Coit DG, Shah MA, Verheij M and Cats A: Treatment of resectable gastric cancer. Therap Adv Gastroenterol 5(1): 49-69, 2012.

35 Yu JW, Zhang P, Wu JG, Wu SH, Li XQ, Wang ST, Lu RQ, Ni $\mathrm{XC}$ and Jiang $\mathrm{BJ}$ : Expressions and clinical significances of CD133 protein and CD133 mRNA in primary lesion of gastric adenocacinoma. J Exper Clin Cancer Res 29: 141, 2010.

36 Sheh A and Fox JG: The role of the gastrointestinal microbiome in Helicobacter pylori pathogenesis. Gut Microbes 4(6): 505-31, 2013.

37 Sato F, Muramatsu S, Tsuchihashi S, Shiragai A, Hiroaka T, Inada T, Kawashima K, Matsuzawa H, Nakamura W, Trucco E and Sacher GA: Radiation effects on cell populations in the intestinal epithelium of mice and its theory. Cell Proliferation 5(3): 227-235, 1972.

38 Ferrand J, Lehours P, Schmid-Alliana A, Megraud F and Varon C: Helicobacter pylori infection of gastrointestinal epithelial cells in vitro induces mesenchymal stem cell migration through an NFkappaB-dependent pathway. PLoS One 6(12): e29007, 2011.

39 Bessede E, Dubus P, Megraud F, Varon C: Helicobacter pylori infection and stem cells at the origin of gastric cancer. Oncogene 34(20): 2547-55, 2015.

40 Yaghoobi M: Bone marrow-derived stem cells in pathogenesis of Helicobacter pylori-associated gastric cancer. Clin Trans1 Gastroenterol 6: e110, 2015.

41 Ding SZ and Zheng PY: Helicobacter pylori infection induced gastric cancer; advance in gastric stem cell research and the remaining challenges. Gut Pathog 4(1): 18, 2012.

42 O'Brien CA, Pollett A, Gallinger S and Dick JE: A human colon cancer cell capable of initiating tumour growth in immunodeficient mice. Nature 445(7123): 106-110, 2007.

43 Oshima A, Hirata N, Ubukata T, Umeda K and Fujimoto I: Evaluation of a mass screening program for stomach cancer with a case-control study design. Int J Cancer 38(6): 829-833, 1986.

44 Fan L, He F, Liu H, Zhu J, Liu Y, Yin Z, Wang L, Guo Y, Wang Z, Yan Q, Huang G: CD133: a potential indicator for differentiation and prognosis of human cholangiocarcinoma. BMC Cancer 11: 320-327, 2011.

45 Immervoll H, Hoem D, Sakariassen PO, Steffensen OJ and Molven A: Expression of the "stem cell marker" CD133 in panceas and pancreatic ductal adenocarcinoma. BMC Cancer 8: 48, 2008.

46 Marzesco AM, Janich P, Wilsch-Brauninger M, Dubreuil V, Langenfeld K, Corbeil D and Huttner WB: Release of extracellular membrane particles carrying the stem cell marker prominin-1 (CD133) from neural progenitors and other epithelial cells. J Cell Sci 118: 2849-2858, 2005. 